\title{
BMJ Open Multicentre validation of a sepsis prediction algorithm using only vital sign data in the emergency department, general ward and ICU
} Qingqing Mao, ${ }^{1}$ Melissa Jay, ${ }^{1}$ Jana L Hoffman, ${ }^{1}$ Jacob Calvert,
Christopher Barton, ${ }^{2}$ David Shimabukuro, ${ }^{3}$ Lisa Shieh, ${ }^{4}$ Uli Chettipally, ${ }^{2,5}$
Grant Fletcher, ${ }^{6}$ Yaniv Kerem, ${ }^{7,8}$ Yifan Zhou, ${ }^{1,9}$ Ritankar Das ${ }^{1}$
To cite: Mao Q, Jay $M$, Hoffman JL, et al. Multicentre validation of a sepsis prediction algorithm using only vital sign data in the emergency department, general ward and ICU. BMJ Open 2018;8:e017833. doi:10.1136/ bmjopen-2017-017833

- Prepublication history and additional material for this paper are available online. To view these files, please visit the journal online (http://dx.doi org/10.1136/bmjopen-2017017833).

Received 18 May 2017 Revised 17 October 2017 Accepted 31 October 2017

Check for updates

For numbered affiliations see end of article.

Correspondence to Dr Jana L Hoffman; jana@dascena.com

\section{ABSTRACT}

Objectives We validate a machine learning-based sepsis-prediction algorithm (InSight) for the detection and prediction of three sepsis-related gold standards, using only six vital signs. We evaluate robustness to missing data, customisation to site-specific data using transfer learning and generalisability to new settings.

Design A machine-learning algorithm with gradient tree boosting. Features for prediction were created from combinations of six vital sign measurements and their changes over time.

Setting A mixed-ward retrospective dataset from the University of California, San Francisco (UCSF) Medical Center (San Francisco, California, USA) as the primary source, an intensive care unit dataset from the Beth Israel Deaconess Medical Center (Boston, Massachusetts, USA) as a transfer-learning source and four additional institutions' datasets to evaluate generalisability. Participants 684443 total encounters, with 90353 encounters from June 2011 to March 2016 at UCSF. Interventions None.

Primary and secondary outcome measures Area under the receiver operating characteristic (AUROC) curve for detection and prediction of sepsis, severe sepsis and septic shock.

Results For detection of sepsis and severe sepsis, InSight achieves an AUROC curve of 0.92 ( $95 \% \mathrm{Cl} 0.90$ to 0.93 ) and 0.87 (95\% Cl 0.86 to 0.88$)$, respectively. Four hours before onset, InSight predicts septic shock with an AUROC of 0.96 (95\% Cl 0.94 to 0.98$)$ and severe sepsis with an AUROC of 0.85 (95\% Cl 0.79 to 0.91$)$.

Conclusions InSight outperforms existing sepsis scoring systems in identifying and predicting sepsis, severe sepsis and septic shock. This is the first sepsis screening system to exceed an AUROC of 0.90 using only vital sign inputs. InSight is robust to missing data, can be customised to novel hospital data using a small fraction of site data and retains strong discrimination across all institutions.

\section{INTRODUCTION}

Sepsis is a major health crisis and one of the leading causes of death in the USA. ${ }^{1}$ Approximately 750000 hospitalised patients
Strengths and limitations of this study

- Machine learning is applied to the detection and prediction of three separate sepsis standards in the emergency department, general ward and intensive care settings.

- Only six commonly measured vital signs are used as input for the algorithm.

- The algorithm is robust to randomly missing data.

- Transfer learning successfully leverages large dataset information to a target dataset.

- The retrospective nature of the study does not predict clinician reaction to information.

are diagnosed with severe sepsis in the USA annually, with an estimated mortality rate of up to one-third. ${ }^{2} 3$ The cost burden of sepsis is disproportionately high, with estimated costs of US $\$ 20.3$ billion annually, or US $\$ 55.6$ million per day in US hospitals. ${ }^{4}$ Additionally, the average hospital stay for sepsis is twice as expensive as other conditions, ${ }^{5}$ and the average incidence of severe sepsis is increasing by approximately $13 \%$ per year. ${ }^{6}$ Early diagnosis and treatment have been shown to reduce mortality and associated costs. $^{7-9}$ Despite clear benefits, early and accurate sepsis detection remains a difficult clinical problem.

Sepsis has been defined as a dysregulated host response to infection. In practice, sepsis can be challenging to recognise because of the heterogeneity of the host response to infection and the diversity of possible infectious insult. Sepsis has been traditionally recognised as two or more systemic inflammatory response syndrome (SIRS) ${ }^{10}$ criteria together with a known or suspected infection; progressing to severe sepsis, in the event of organ dysfunction and finally to septic shock, which additionally includes refractory 
hypotension. ${ }^{10}$ However, ongoing debates over sepsis definitions and clinical criteria, as evidenced by the recent proposed redefinitions of sepsis, ${ }^{11}$ underscore a fundamental difficulty in the identification and accurate diagnosis of sepsis.

Various rule-based disease-severity scoring systems are widely used in hospitals in an attempt to identify patients with sepsis. These scores, such as the Modified Early Warning Score (MEWS), ${ }^{12}$ the SIRS criteria ${ }^{13}$ and the Sequential Organ Failure Assessment (SOFA), ${ }^{14}$ are manually tabulated at the bedside and lack accuracy in sepsis diagnosis. However, the increasing prevalence of electronic health records (EHRs) in clinical settings provides an opportunity for enhanced patient monitoring and increased early detection of sepsis.

This study validates a machine-learning algorithm, InSight, which uses only six vital signs taken directly from the EHR, in the detection and prediction of sepsis, severe sepsis and septic shock in a mixed-ward population at the University of California, San Francisco (UCSF). We investigate the effects of induced data sparsity on InSight performance and compare all results with other scores that are commonly used in the clinical setting for the detection and prediction of sepsis. We additionally train and test the algorithm for severe sepsis detection on data from Stanford Medical Center and three community hospitals in order to better estimate its expected clinical performance. Furthermore, we apply a transfer-learning scheme to customise a Multiparameter Intelligent Monitoring in Intensive Care (MIMIC)-III-trained algorithm to the UCSF patient population using a minimal amount of UCSF-specific data.

\section{METHODS}

\section{Datasets}

We used a dataset provided by the UCSF Medical Center representing patient stays from June 2011 to March 2016 in all experiments. The UCSF dataset contains 17467987 hospital encounters, including inpatient and outpatient visits to all units within the UCSF medical system. The data were deidentified to comply with the Health Insurance Portability and Accountability Act (HIPAA) Privacy Rule. For transfer learning, we used the MIMIC-III v1.3 dataset, compiled from the Beth Israel Deaconess Medical Center (BIDMC) in Boston, Massachusetts, between 2001 and 2012, composed of 61532 intensive care unit (ICU) stays. ${ }^{15}$ This database is a publicly available database constructed by researchers at Massachusetts Institute of Technology's Laboratory for Computational Physiology, and the data were also deidentified in compliance with HIPAA. Additionally, we trained and tested the algorithm for severe sepsis detection on data from Stanford Medical Center (Stanford, California, USA), ${ }^{16}$ Oroville Hospital (Oroville, California, USA), Bakersfield Heart Hospital (BHH; Bakersfield, California, USA) and Cape Regional Medical Center (CRMC; Cape May Courthouse, New Jersey, USA). Details on these datasets are included in the online supplementary tables 1 and 2. Data collection for all datasets did not impact patient safety. Therefore, this study constitutes non-human subjects research, which does not require institutional review board approval.

\section{Data extraction and imputation}

The data were provided in the form of comma-separated-value files and stored in a PostgreSQL ${ }^{17}$ database. Custom Structure Query Language queries were written to extract measurements and patient outcomes of interest. The measurement files were then binned by hour for each patient. To be included, patients were required to have at least one of each type of measurement recorded during the encounter. If a patient did not have a measurement in a given hour, the missing measurement was filled in using carry-forward imputation. This imputation method applied the patient's last measured value to the following hour (a causal procedure). In the case of multiple measurements within an hour, the mean was calculated and used in place of an individual measurement. After the data were processed and imputed in Python, ${ }^{18}$ they were used to train the InSight classifier and test its predictions at sepsis onset and at fixed time points prior to onset.

\section{Gold standards}

In this study, we tested InSight's performance according to various gold standards (clinical indications). We investigated InSight's ability to predict and detect sepsis, severe sepsis and septic shock. Further, we compared InSight's performance to SIRS, MEWS and SOFA, for each of the following gold standards. For training and testing the algorithm, we conservatively identified each septic condition by requiring that the International Classification of Diseases (ICD) 9 code corresponding to the diagnosis was coded for each positive case, in addition to meeting the clinical requirements for the definition of each septic standard as defined below.

\section{Sepsis}

The sepsis gold standard was determined using the 2001 consensus sepsis definition ${ }^{10}$ : 'the presence of two or more SIRS criteria paired with a suspicion of infection'. To identify a case as positive for sepsis, we required ICD-9 code 995.91. The onset time was defined as the first time two or more SIRS criteria were met within the same hour. SIRS criteria are defined as:

- heart rate $>90$ beats per minute

- body temperature $>38^{\circ} \mathrm{C}$ or $<36^{\circ} \mathrm{C}$

- respiratory rate $>20$ breaths $/ \mathrm{min}$ or $\mathrm{PaCO}_{2}$ (alveolar carbon dioxide tension) $<32 \mathrm{~mm} \mathrm{Hg}$

- white cell count $>12 \times 109$ cells $/ \mathrm{L}$ or $<4$ x 109 cells $/ \mathrm{L} .{ }^{10}$

Severe sepsis

The severe sepsis gold standard used the definition of severe sepsis as 'organ dysfunction caused by sepsis' which can be represented by one or more of the criteria below, and identified for patients with the severe sepsis ICD-9 code 995.92. We assigned the severe sepsis onset time to 
be the first instance during which two SIRS criteria, as described in the Sepsis section, and one of the following organ dysfunction criteria were met within the same hour:

- lactate $>2 \mathrm{mmol} / \mathrm{L}$

- systolic blood pressure $<90 \mathrm{~mm} \mathrm{Hg}$

- urine output $<0.5 \mathrm{~mL} / \mathrm{kg}$, over 2 hours, prior to organ dysfunction after fluid resuscitation

- creatinine $>2 \mathrm{mg} / \mathrm{dL}$ without renal insufficiency or chronic dialysis

- bilirubin $>2 \mathrm{mg} / \mathrm{dL}$ without having liver disease or cirrhosis

- platelet count $<100000 \mu \mathrm{L}$

- international normalised ratio $>1.5$

- arterial oxygen tension $\left(\mathrm{PaO}_{2}\right) /$ fractional inspired oxygen $\left(\mathrm{FiO}_{2}\right)<200$ in addition to pneumonia, $<250$ with acute kidney injury but without pneumonia.

\section{Septic shock}

We identified as positive cases for septic shock those patients who received the septic shock ICD-9 code 785.52 and additionally demonstrated the following conditions:

- systolic blood pressure of $<90 \mathrm{~mm} \mathrm{Hg}$, defined as hypotension, for at least $30 \mathrm{~min}$ and

- who were resuscitated with $\geq 20 \mathrm{~mL} / \mathrm{kg}$ over a 24 -hour period, or

- who received $\geq 1200 \mathrm{~mL}$ in total fluids. ${ }^{19}$

The onset time was defined as the first hour when either the hypotension or the fluid resuscitation criterion was met.

\section{Calculating comparators}

We compared InSight predictions for each gold standard to three common patient deterioration scoring systems: SIRS, SOFA and MEWS. Area under the receiver operating characteristic (AUROC) curve, sensitivity and specificity were compared across all prediction models. The SIRS criteria, as explained in the sepsis definition, were evaluated independently of the suspicion of infection. To calculate the SOFA score, we collected each patient's $\mathrm{PaO}_{2} / \mathrm{FiO}_{2}$, Glasgow Coma Score, mean arterial blood pressure or administration of vasopressors, bilirubin level, platelet counts and creatinine level. Each of the listed measurements is associated with a SOFA score of $1-4$, based on severity level, as described by Vincent et al. ${ }^{14}$ After receiving a score for each of the six organ dysfunction categories, the overall SOFA score was computed as the sum of the category scores and used as a comparator to InSight. Finally, the MEWS score, which ranges from 0 (normal) to 14 (high risk of deterioration), was determined by tabulating subscores for heart rate, systolic blood pressure, respiratory rate, temperature and Glasgow Coma Score. We used the subscoring system presented in Fullerton $e t a l^{20}$ to compute each patient's MEWS score.

\section{Measurements and patient inclusion}

In order to generate InSight scores, patient data were analysed from each of the following six clinical vital sign measurements: systolic blood pressure, diastolic blood pressure, heart rate, respiratory rate, peripheral capillary oxygen saturation and temperature. We used only vital signs, which are frequently available and routinely taken in the ICU, emergency department (ED) and floor units. Patient data were used from the course of a patient's hospital encounter, regardless of the unit the patient was in when the data were collected.

All patients over the age of 18 years were considered for this study. For a given encounter, if the patient was admitted to the hospital from the ED, the start of the ED visit is where the analysis began. Patients in our final datasets were required to have at least one measurement for each of the six vital signs. In order to ensure enough data to accurately characterise sepsis predictions at 4 hours preonset, we further limited the study group to exclude patients whose septic condition onset time was within 7 hours after the start of their record, which was either the time of admission to the hospital or the start of their ED visit; the latter was applicable only if the patient was admitted through the ED. A smaller window to sepsis onset time would have resulted in insufficient testing data to make 4-hour prediction possible in some cases, which would inappropriately affect performance metrics such as sensitivity and specificity. Patients with sepsis onset after 2000 hours postadmission were also excluded, to limit the data-analysis matrix size. The final UCSF dataset included 90353 patients (figure 1), and the MIMIC-III dataset contained 21604 patients, following the same inclusion criteria. Inclusion criteria and final inclusion numbers for the Stanford, Oroville, BHH and CRMC datasets are included in online supplementary table 1 .

After patient exclusion, our final group of UCSF patients was composed of $55 \%$ women and $45 \%$ men with a median age of 55 years. The median hospital length of stay was 4 days, IQR 2-6. Of the 90353 patients, 1179 were found to have sepsis (1.30\%), 349 were identified as having severe sepsis without shock $(0.39 \%)$ and 614 were determined to have septic shock $(0.68 \%)$. The in-hospital mortality rate was $1.42 \%$. Patient encounters spanned a variety of wards. The most common units represented in our study were perioperative care, the ED, the neurosciences department and cardiovascular and thoracic transitional care. In the MIMIC-III dataset, approximately $44 \%$ of patients were women and $56 \%$ were men. Stays were typically shorter in this dataset, since each encounter included only an ICU stay. The median length of stay was 2 days. Furthermore, due to the nature of intensive care, there was a higher prevalence of sepsis $(1.91 \%)$, severe sepsis $(2.82 \%)$ and septic shock $(4.36 \%)$. A full summary of baseline characteristics for both datasets is presented in table 1. Full demographic information for the Stanford, Oroville, BHH and CRMC datasets is provided in online supplementary table 2.

\section{Feature construction}

We minimally processed raw vital sign data to generate features. Following EHR data extraction and imputation, we obtained 3-hourly values for each of the six 


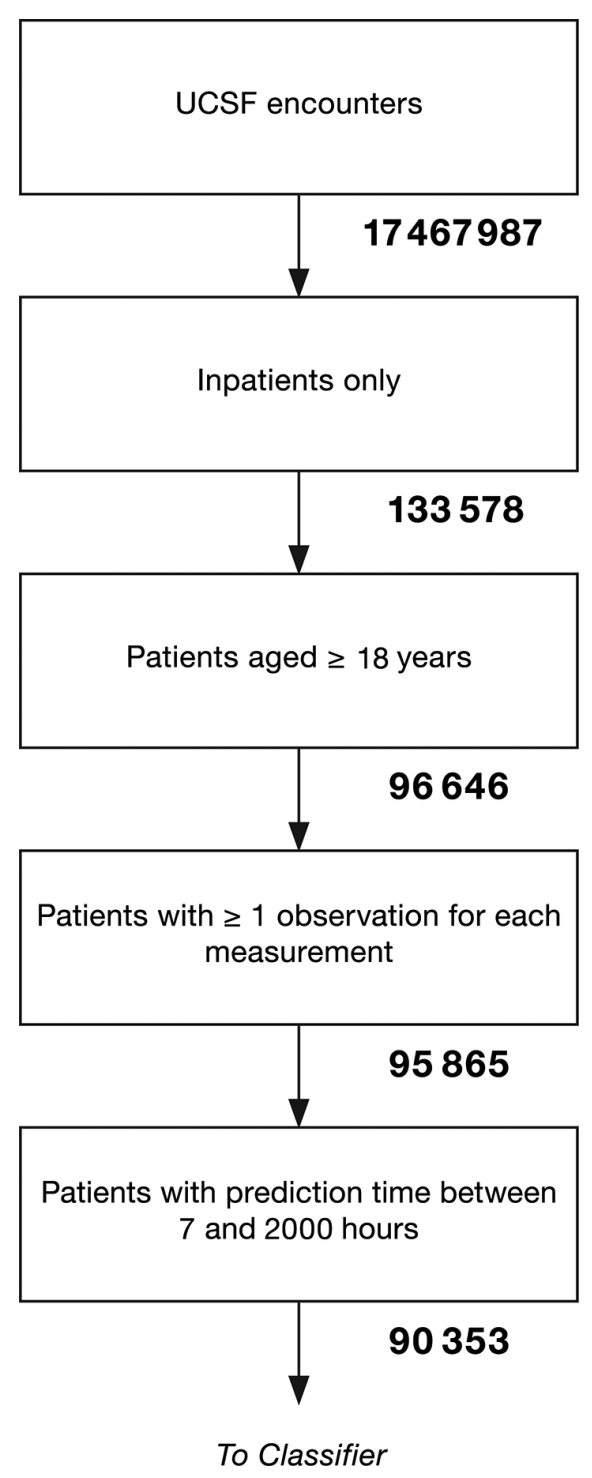

Figure 1 Patient inclusion flow diagram for the UCSF dataset. UCSF, University of California, San Francisco.

vital sign measurement channels from that hour, the hour prior and 2 hours prior. We also calculated two difference values between the current hour and the prior hour, and between the prior hour and the hour before that. We concatenated these five values from each vital sign into a causal feature vector $\mathbf{x}$ with 30 elements (five values from each of the six measurement channels).

\section{Machine learning}

We used gradient tree boosting to construct our classifier. Gradient tree boosting is an ensemble technique which combines the results from multiple weak decision trees in an iterative fashion. Each decision tree was built by discretising features into two categories. For example, one node of the decision tree might have stratified a patient based on whether their respiratory rate was greater than 20 breaths per minute, or not. Depending on the answer for a given patient, a second, third and so on, vital sign may be checked. A risk score was generated for the patient based on their path along the decision tree. We limited each tree to split no more than six times; no more than 1000 trees were aggregated in the iteration through gradient boosting to generate a robust risk score. Training was performed separately for each distinct task and prediction window, and observations were accordingly labelled positive for model fitting for each specific prediction task. Patient measurements were not used after the onset of a positive clinical indication.

We performed 10-fold cross-validation to validate InSight's performance and minimise potential model overfit. We randomly split the UCSF dataset into a training set, composed of $80 \%$ of UCSF's encounters, and an independent test set with the remaining $20 \%$ of encounters. Of the training set, data were divided into 10 groups, nine of which were used to train InSight, and one of which was used to validate. After cycling through all combinations of training and validation sets, we then tested each of the 10 models on the independent test set. Mean performance metrics were calculated based on these 10 models. For severe sepsis detection at the time of onset on each of Stanford, Oroville, BHH and CRMC datasets, we performed fourfold cross-validation of the model.

Additionally, we trained and validated InSight's performance in identifying sepsis, severe sepsis and septic shock after removing all features which were used in our gold standard definitions for each condition. This resulted in the removal of vital sign SIRS criteria measurements for sepsis and severe sepsis predictions, and the removal of systolic and diastolic blood pressure measurements for septic shock. We also trained and validated the algorithm for each of the three gold standards for randomly selected, upsampled and downsampled subpopulations with positive class prevalence between $0 \%$ and $100 \%$.

\section{Missing data}

After assessing InSight's performance on complete datasets, we used a random deletion process to simulate the algorithm's robustness to missing measurements. Individual measurements from the test set were deleted according to a probability of deletion, $\mathrm{P}$. We set $\mathrm{P}=(0$, $0.1,0.2,0.4$ and 0.6 ) for each of our missing data experiments and tested the InSight algorithm on the sparse datasets.

\section{Transfer learning}

To evaluate InSight's performance on a minimal amount of UCSF data, we used a transfer-learning approach. ${ }^{21}$ There are clear dissimilarities in patient demographics, clinical characteristics and average measurement frequencies between the UCSF and MIMIC-III datasets (see table 1). Partially this is because the UCSF data involves a variety of hospital wards, whereas the MIMIC-III dataset provides only measurements taken in the ICU. We sought to determine improved performance 
Table 1 Demographic and clinical characteristics for UCSF patient population analysed ( $n=90353)$ and MIMIC-III patient population analysed $(n=21604)$

\begin{tabular}{llcc}
\hline & & UCSF & MIMIC-III \\
\cline { 3 - 4 } Demographic overview & Characteristic & Count (\%) & Count (\%) \\
\hline Gender & Female & $49763(55.08)$ & $9499(43.97)$ \\
& Male & $40590(44.92)$ & $12105(56.03)$ \\
Age & $18-29$ & $10652(11.79)$ & $978(4.53)$ \\
UCSF: median 55, IQR 38-67 & $30-39$ & $14202(15.72)$ & $1114(5.16)$ \\
MIMIC-III: median 65, IQR 53-77 & $40-49$ & $11888(13.16)$ & $2112(9.78)$ \\
& $50-59$ & $16856(18.66)$ & $3880(17.96)$ \\
& $60-69$ & $19056(21.09)$ & $4906(22.71)$ \\
Length of stay (days) & $70+$ & $17699(19.59)$ & $8614(39.87)$ \\
UCSF: median 4, IQR 2-6 & $0-2$ & $28258(31.26)$ & $11054(51.17)$ \\
MIMIC-III: median 2, IQR 2-4 & $3-5$ & $35128(38.88)$ & $7004(32.42)$ \\
& $6-8$ & $12664(14.02)$ & $1673(7.74)$ \\
& $9-11$ & $4934(5.46)$ & $734(3.40)$ \\
Death during hospital stay & $12+$ & $9369(10.37)$ & $1139(5.27)$ \\
& Yes & $1279(1.42)$ & $1328(6.15)$ \\
ICD-9 code & No & $89074(98.58)$ & $20276(93.85)$ \\
& Sepsis & $1179(1.30)$ & $413(1.91)$ \\
& Severe Sepsis & $349(0.39)$ & $609(2.82)$ \\
& Septic Shock & $614(0.68)$ & $943(4.36)$ \\
\hline
\end{tabular}

ICD, International Classification of Diseases; IQR, Interquartile Range; MIMIC, Multiparameter Intelligent Monitoring in Intensive Care; UCSF, University of California, San Francisco.

metrics on the UCSF target dataset, when the algorithm is primarily trained on MIMIC-III. Using MIMIC-III data as the source, and UCSF as the target, we trained the InSight classifier according to the severe sepsis gold standard. Variable amounts of UCSF training data were incrementally added to the MIMIC-III training dataset, and the resulting model was then validated on the separate UCSF test dataset. Specifically, we left $50 \%$ of the UCSF patients as test data, and we randomly selected different fractions of the remaining UCSF data and combined them with the entire MIMIC-III dataset as the training data. For each fraction used, we trained
100 models with different random relative weights on the UCSF and MIMIC-III training data. Then, the mean and SD of AUROC values for each of these models were calculated on 20 randomly sampled sets, and the model with the highest mean AUROC value among these 100 was used.

\section{RESULTS}

InSight's performance on the UCSF data set with respect to MEWS, SOFA and SIRS is summarised in figure 2A-C. Figure 2A-C demonstrate InSight's ability to accurately


Figure 2 ROC curves for InSight and common scoring systems at the time of (A) sepsis onset, (B) severe sepsis onset and (C) 4 hours before septic shock onset. MEWS, Modified Early Warning Score; ROC, receiver operating characteristic; SIRS, systemic inflammatory response syndrome; SOFA, Sequential Organ Failure Assessment. 
detect the onset of sepsis and severe sepsis, and to accurately predict septic shock 4 hours prior to onset, compared with the performance of common sepsis scoring systems. Each figure presents InSight's receiver operating characteristic (ROC) curve together with the ROC curves for MEWS, SOFA and SIRS. InSight achieves an AUROC curve for sepsis onset of 0.92 (95\% CI 0.90 to 0.93 ), for severe sepsis onset of 0.87 (95\% CI 0.86 to 0.88 ) and for septic shock of 0.99 (95\% CI 0.9991 to 0.9994$)$ compared with SIRS, which demonstrates an AUROC of $0.75,0.72$ and 0.84 , respectively. Even when all gold standard involved measurements were removed from model training, InSight continued to demonstrate improved accuracy over SIRS, MEWS and SOFA, with AUROC values of 0.84 (95\% CI 0.83 to 0.85 ) for sepsis onset, 0.80 (95\% CI 0.79 to 0.81 ) for severe sepsis onset and 0.96 (95\% CI 0.96 to 0.97 ) for septic shock onset.

Comparing InSight's performance across the three sepsis-related gold standards, it is clear that the septic shock criteria are relatively less challenging to anticipate, as its 4-hour prediction metrics are stronger than those for the detection of both sepsis and severe sepsis. Accordingly, we display the 4 hours prior to onset prediction case for septic shock (figure 2C), where existing tools fail to adequately meet prediction standards relevant for sound clinical use. Four hours in advance of septic shock onset, InSight achieved an AUROC of 0.96 (95\% CI 0.94 to 0.98 ). The resulting confusion matrix from the 10-fold cross-validation of InSight can be found in online supplementary tables 3 and 4.

Additional comparison metrics at the time of detection for each gold standard are available in table 2. In order to compare the specificities from each gold standard, we fixed sensitivities near 0.80 ; that is, we fixed a point on the ROC curve (ie, set a specific threshold) after model development and tested algorithm performance under the chosen conditions in order to present data as consistently as possible. We similarly fixed specificities near 0.80 in order to compare sensitivities. Across all gold standards, a sensitivity of 0.80 results in a high specificity for InSight; however, the sensitivities for MEWS, SOFA and SIRS are significantly lower. Notably, at 0.80 sensitivity, InSight achieves a specificity of 0.95 for sepsis, 0.84 for severe sepsis and 0.99 for septic shock detection.

In addition to InSight's ability to detect sepsis, severe sepsis and septic shock, figure $3 \mathrm{~A}$ illustrates the ROC of severe sepsis detection and prediction 4 hours prior to severe sepsis onset. Even 4 hours in advance, the InSight severe sepsis AUROC is 0.85 (95\% CI 0.79 to 0.91 ), which is significantly higher than the onset time SIRS AUROC of 0.75 AUROC. Figure 3B summarises InSight's predictive advantage, using the severe sepsis gold standard, over MEWS, SOFA and SIRS at the same time points in the hours leading up to onset. InSight maintains a high AUROC in the continuum up to 4 hours preceding severe sepsis onset. InSight's predictions 4 hours in advance produce a sensitivity and specificity that are greater than the at-onset time sensitivity and specificity of each MEWS, SOFA and SIRS (table 2, figure 3B).

In order to determine the generalisability of the algorithm to different settings, we tested InSight on additional patient datasets from four distinct hospitals. For severe sepsis detection at the time of onset, InSight achieved AUROC over 0.92 on patients from Stanford, Oroville Hospital, Bakersfield Heart Health and CRMC (table 3). ROC curves and comparisons to alternate sepsis classification systems on these datasets are presented in the online supplementary tables 5-8 and figures 1 and 2). InSight AUROC values exceed those of the MEWS, SIRS, quick SOFA (qSOFA) and SOFA scores on the same datasets for severe sepsis detection at the time of onset.

We ranked feature importance for the classifiers developed in this experiment and determined that systolic blood pressure at the time of prediction was consistently the most important feature in making accurate model

Table 2 Performance metrics for three sepsis gold standards at the time of onset (0 hour), with sensitivities fixed at or near 0.80 in the first instance and specificities fixed at or near 0.80 in the second instance

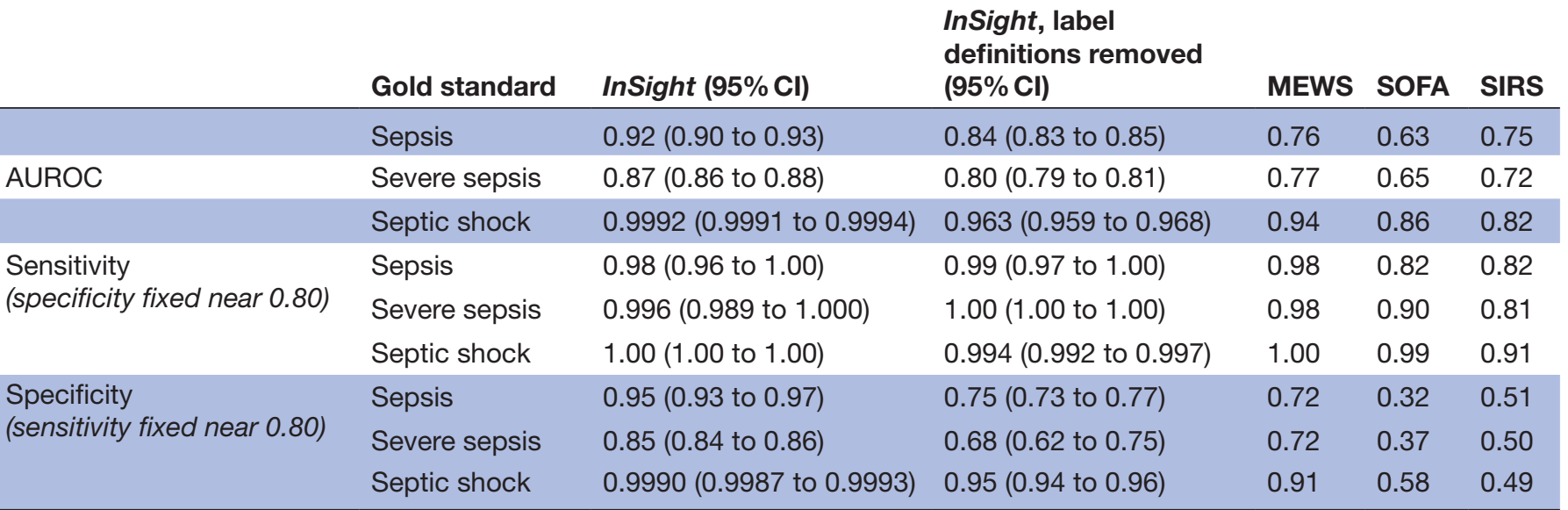

AUROC, area under the receiver operating characteristic; Cl, Confidence Interval; MEWS, Modified Early Warning Score; SIRS, systemic inflammatory response syndrome; SOFA, Sequential Organ Failure Assessment. 

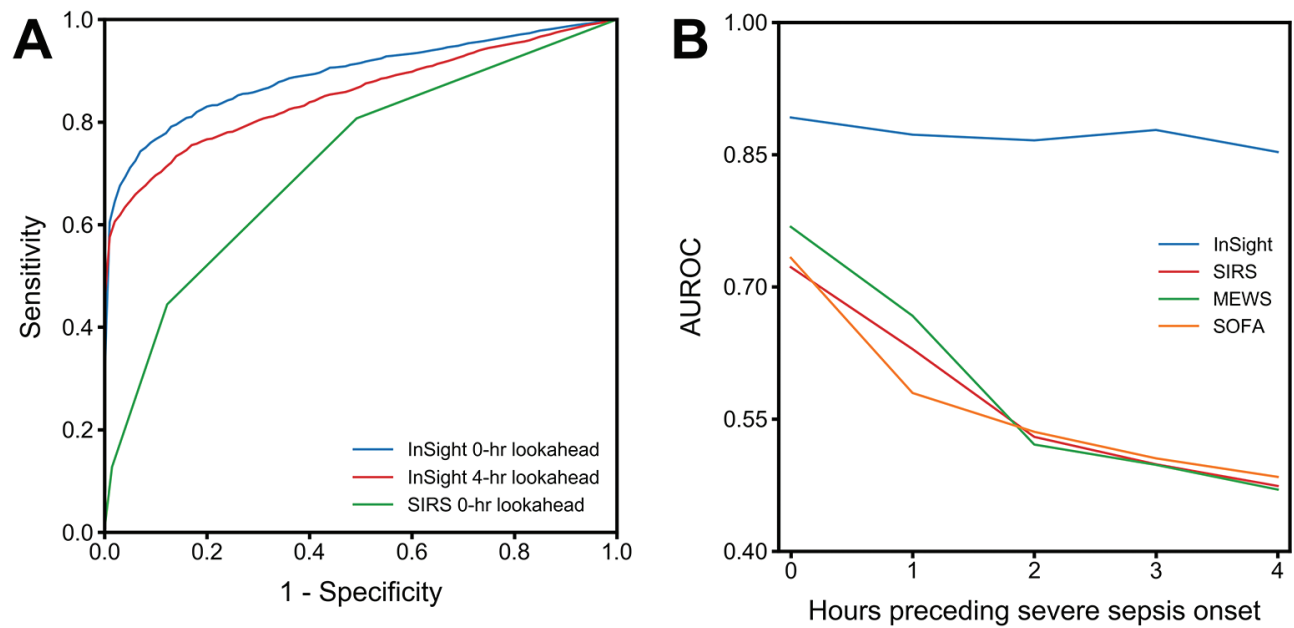

Figure 3 (A) ROC detection (0 hour, blue) and prediction (4 hours prior to onset, red) curves using InSight and ROC detection (0 hour, green) curve for SIRS, with the severe sepsis gold standard. (B) Predictive performance of InSight and comparators, using the severe sepsis gold standard, as a function of time prior to onset. AUROC, area under the receiver operating characteristic; ROC, receiver operating characteristic; MEWS, Modified Early Warning Score; SIRS, systemic inflammatory response syndrome; SOFA, Sequential Organ Failure Assessment.

predictions. The relative importance of other features varied significantly based on the specific prediction task.

In our second set of experiments, we validated InSight's performance in the presence of missing data. We tested InSight's ability to detect severe sepsis at the time of onset with various rates of data dropout. Table 4 presents the results of these experiments. After randomly deleting data from the test set with a probability of 0.10 , InSight's AUROC for severe sepsis detection is 0.82 . Dropping approximately $60 \%$ of the test set measurements results in an AUROC of 0.75, demonstrating InSight's robustness to missing data. Of note, the AUROC of InSight at $60 \%$ data dropout achieves slightly better performance than SIRS with no missing data. Further, our experiments on applying InSight to upsampled and downsampled sets showed that AUROC was largest when the set was chosen such that around half the patients met the gold standard. Moving lower on prevalence from $50 \%$ down to $0 \%$, the AUROC values were only slightly lower while they dropped steeply when moving higher on prevalence from $50 \%$ up to $100 \%$ (a clinically unrealistic range).

\section{Transfer learning}

InSight is flexible by design and can be easily trained on an appropriate retrospective dataset before being applied to a new patient population. However, sufficient historical patient data are not always available for training on the target population. We evaluated InSight's performance when trained on a mixture of the MIMIC-III data together with increasing amounts of UCSF training data, and then tested on a separate hold-out UCSF patient population using transfer learning. In figure 4, we show that the performance of the algorithm improves as the fraction of UCSF target population data used in training increases.

Feature importance was quite stable across transfer-learning experiments, with systolic blood pressure measurements consistently playing an important role. Systolic blood pressure at 2 hours before onset, at the time of onset and at 1 hour before onset, in that order, were the most important features for accurate prediction in all tasks. Heart rate and diastolic blood pressure at the time of onset were consistently the fourth and fifth most important features, though order of importance of the two features varied between tasks.

\section{DISCUSSION}

We have validated the machine-learning algorithm, InSight, on the mixed-ward data of UCSF, which includes patients from the ED and floor units as well as the ICU, with

Table 3 Algorithm performance for severe sepsis detection at the time of onset

\begin{tabular}{|c|c|c|c|c|}
\hline & Stanford & Oroville & ВHH & CRMC \\
\hline Sensitivity & 0.798 & 0.806 & 0.875 & 0.802 \\
\hline Accuracy & 0.900 & 0.971 & 0.963 & 0.931 \\
\hline LR+ & 8.253 & 77.92 & 58.94 & 16.85 \\
\hline
\end{tabular}

AUROC, area under the receiver operating characteristic; BHH, Bakersfield Heart Hospital; Cl, Confidence Interval; CRMC, Cape Regional Medical Center; LR, likelihood ratio. 
Table 4 InSight's severe sepsis screening performance at the time of onset in the presence of data sparsity compared with SIRS with a full data complement

\begin{tabular}{lllllll}
\hline & \multicolumn{2}{l}{ InSight } & & & & SIRS \\
\cline { 2 - 6 } Data missing (\%) & $\mathbf{0}$ & $\mathbf{1 0}$ & $\mathbf{2 0}$ & $\mathbf{4 0}$ & $\mathbf{6 0}$ & $\mathbf{0}$ \\
\hline AUROC & 0.90 & 0.82 & 0.79 & 0.76 & 0.75 & 0.72 \\
Sensitivity & 0.80 & 0.80 & 0.80 & 0.80 & 0.80 & 0.80 \\
Specificity & 0.84 & 0.66 & 0.57 & 0.50 & 0.49 & 0.51 \\
\hline
\end{tabular}

AUROC, area under the receiver operating characteristic; SIRS, systemic inflammatory response syndrome.

varying types and frequencies of patient measurements. InSight outperformed commonly used disease severity scores such as SIRS, MEWS and SOFA for the screening of sepsis, severe sepsis and septic shock (figure 2). These results, shown in table 2, confirm InSight's strength in predicting these sepsis-related gold-standard outcomes. The algorithm's strong performance across the academic and community hospital data used in this study suggests potential strong performance in a variety of future clinical settings.

To the authors' knowledge, InSight is the first sepsis screening system to meet or exceed an AUROC of 0.90 using only vital sign inputs, on each of the sepsis gold standards evaluated in this study. Additionally, InSight provides predictive capabilities in advance of sepsis onset, aided by the analysis of trends and correlations between vital sign measurements. This advantage is apparent in the comparison with SIRS made in figure 3A. Up to 4 hours prior to severe sepsis onset, InSight maintains a high AUROC above 0.85 (figure 3 ). This advance warning of patients

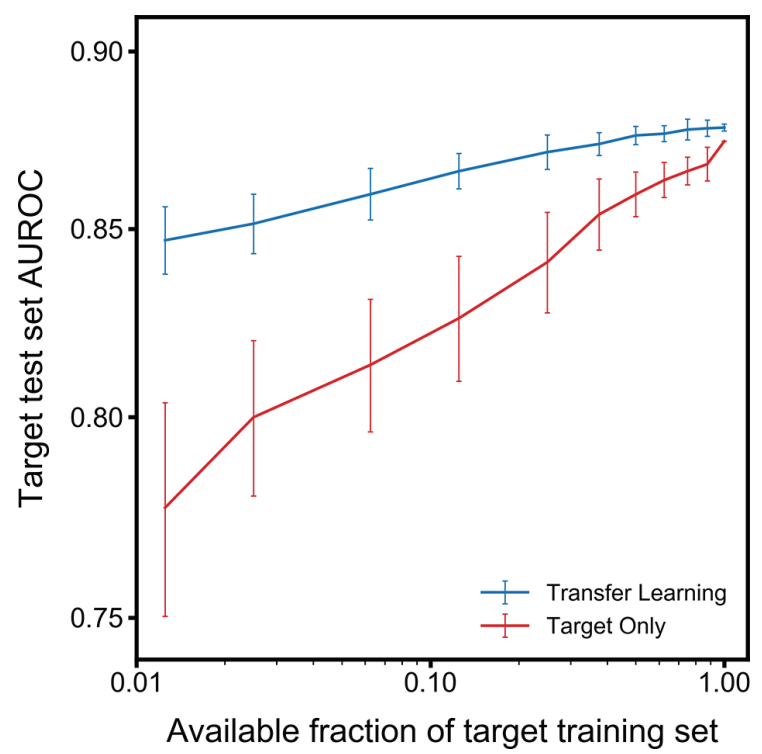

Figure 4 Learning curves (mean AUROC on the UCSF target dataset) with increasing number of target training examples. Error bars represent the Standard Deviation. When data availability of the target set is low, target-only training exhibits lower AUROC values and high variability. AUROC, area under the receiver operating characteristic; UCSF, University of California, San Francisco. trending towards severe sepsis could extend the window for meaningful clinical intervention.

InSight uses only six common vital signs derived from a patient's EHR to detect sepsis onset, as well as to predict those patients who are most at risk for developing sepsis. The decreased performance of InSight for recognition of severe sepsis relative to sepsis onset may be in part because the organ failure characteristic of severe sepsis is more easily recognisable through laboratory tests for organ function. Because we have not incorporated metabolic function panels in this validation of InSight, the detection of organ failure using only six common vital signs may be more difficult. In practice, InSight is adaptable to different inputs and is able to incorporate laboratory results as they become available. Inclusion of these results may well increase the performance of InSight for the detection and prediction of severe sepsis. However, in this work, we have chosen to benchmark the performance of InSight using only six commonly measured vital signs. The ordering of metabolic panel laboratory tests are often predicated on clinician suspicion of severe sepsis, and therefore, early or developing cases may be missed. Additionally, because these vital sign inputs do not require time-dependent laboratory results or additional manual data entry, surveillance by InSight is frequent, and as a result, sepsis conditions are detected in a more timely manner. Minimal data requirements also lighten the burden of implementation in a clinical setting and broaden the potential clinical applications of InSight.

Although InSight uses only a handful of clinical variables, it maintains a high level of performance in experiments with randomly missing data. We demonstrate in table 4 that for the detection of severe sepsis, even with up to $60 \%$ of randomised test patient data missing, InSight still achieves slightly better performance to SIRS calculated with complete data availability.

Additionally, we have investigated the customisability of InSight to local hospital demographics and measurements. The incorporation of site-specific data into the training set using transfer learning improves performance on test sets, over that of a training set composed entirely of an independent population. This indicates that it may be possible to adequately train InSight for use in a new clinical setting, while still predominantly using existing retrospective data from other institutions. Further, the results of our 
upsampling and downsampling experiments indicate that InSight is likely to only be slightly less effective (in AUROC terms) in settings with lower prevalence of sepsis, severe sepsis or septic shock, than UCSF or slightly more effective if the prevalence is higher than UCSF.

Our previous studies, performed on earlier versions of the model, have investigated InSight applied to individual sepsis standards such as the SIRS standard for sepsis, ${ }^{22}$ severe sepsis ${ }^{23}$ and septic shock, ${ }^{24}$ on the MIMIC retrospective datasets. We have also developed a related algorithm to detect patient stability ${ }^{25}$ and predict mortality. ${ }^{26}{ }^{27}$ However, this study, which evaluates a significantly improved algorithm, is the first to apply InSight to all three standard sepsis definitions simultaneously, and to validate the algorithm on a mixed ward population, including ED, ICU and floor wards from UCSF. This study is also the first to use only six minimal vital signs, without using a mental status evaluation such as Glasgow Coma Score, or even age, in the detection and prediction of those sepsis standards.

The separate models trained for each gold standard and prediction window in this study further demonstrate the potential clinical utility of machine-learning methods. In addition to training on a specific patient population, machine-learning methods can allow for the development of prediction models which are tailored to a hospital's unique needs, data availability and existing workflow practices. Any one of the models developed in this study could be independently deployed in a clinical setting; choice of model deployment would be contingent on the needs of a particular hospital, and the expected trade-off in performance for different model choices. Additionally, this study demonstrates the adaptability of the machinelearning algorithm to an entirely new patient dataset with markedly different demographics and outcomes through both site-specific retraining and transfer-learning techniques.

\section{Limitations}

While we incorporated data from multiple institutions, we cannot claim generalisability of our results to other populations on the basis of this study alone. However, we are aided by the minimality of data used to make predictions. As InSight requires only six of the most basic and widely available clinical measurements, it is likely that it will perform similarly in other settings if vital sign data is available. The gold standard references we use to determine sepsis, severe sepsis and septic shock rely on ICD-9 codes from the hospital database. This standard potentially limits our ability to capture all patients with sepsis in the dataset, should any have been undiagnosed or improperly recorded. The administrative coding procedures may vary by hospital and do not always precisely reproduce results from manual chart review for sepsis diagnosis, although ICD-9 codes have been previously validated for accuracy in the detection of severe sepsis. ${ }^{28}$ The vital sign measurements abstracted from the EHR are basic measurements routinely collected from all patients regardless of the diagnosis and independent of physician judgement, and therefore this input to InSight is not dependent on the time of clinical diagnosis. However, the ordering of laboratory tests is contingent on physician suspicion, and the timing of these inputs may reflect clinician judgement rather than true onset time, potentially limiting the accuracy of our analysis.

While the imputation and averaging performed before feature construction eliminated some information about sampling frequency, these methods do not remove all non-physiological information inherent to our system. Further, imputation of the most recently available past measurement may artificially alter the rate of the temporal changes in patient vital signs that we incorporate into feature vectors, which may in turn affect risk predictions. Averaging multiple patient measurements may similarly remove informative variation in vital signs.

It is important to note that we designed the study as a classification task rather than a time-to-event modelling experiment because the former is significantly more common in the literature..$^{29-32}$ The alternative would not allow for the use of an established, standard set of performance metrics such as AUROC and specificity without custom modification, and would make it more difficult to compare the present study to prior work in the field. This study was conducted retrospectively, and so we are unable to make claims regarding performance in a prospective setting, which involves the interpretation and use of InSight's predictions by clinicians. Additionally, our inclusion criteria requiring at least 7 hours of patient data preceding sepsis onset also limit generalisability to a clinical setting where the predictor would receive data in real time. Algorithm performance in a clinical setting may reasonably be expected to be lower than its retrospective performance in this study. Finally, our random deletion of data is not necessarily representative of data scarcity as it would occur in clinical settings where the rate of missing measurements would depend on the standard rate of data collection, which can vary widely, especially between the ED, general ward and ICU. We intend to evaluate these algorithms in prospective clinical studies in future work.

\section{CONCLUSIONS}

We have validated the machine-learning algorithm, InSight, in a multicentre study including a mixed-ward population from UCSF and an ICU population from BIDMC. InSight provides high sensitivity and specificity for the detection and prediction of sepsis, severe sepsis and septic shock using the analysis of only six common vital signs taken from the EHR. InSight outperforms scoring 
systems in current use for the detection of sepsis, is robust to a significant amount of missing patient data and can be customised to novel sites using a limited amount of site-specific data. Our results indicate that InSight outperforms tools currently used for sepsis detection and prediction which may lead to improvements in sepsis-related patient outcomes.

\section{Author affiliations}

${ }^{1}$ Dascena Inc., Hayward, California, USA

${ }^{2}$ Department of Emergency Medicine, University of California San Francisco, San Francisco, California, USA

${ }^{3}$ Department of Anesthesia and Perioperative Care, University of California San Francisco, San Francisco, California, USA

${ }^{4}$ Department of Medicine, Stanford University School of Medicine, Stanford, California, USA

${ }^{5}$ Kaiser Permanente South San Francisco Medical Center, South San Francisco, California, USA

${ }^{6}$ Division of Internal Medicine, University of Washington School of Medicine, Seattle, Washington, USA

${ }^{7}$ Department of Clinical Informatics, Stanford University School of Medicine, Stanford, California, USA

${ }^{8}$ Department of Emergency Medicine, Kaiser Permanente Redwood City Medical Center, Redwood City, California, USA

${ }^{9}$ Department of Statistics, University of California Berkeley, Berkeley, California, USA

Acknowledgements We acknowledge the assistance of Siddharth Gampa, Anna Lynn-Palevsky, and Emily Huynh for editing contributions. We thank Dr Hamid Mohamadlou and Dr Thomas Desautels for contributions to the development of the machine-learning algorithm, InSight. We also acknowledge Zirui Jiang for valuable computational assistance. We gratefully thank Matthew N Fine, MD, Dr Andrea McCoy, and Chris Maupin, RN, for access to patient datasets. We also thank Dr Gomathi Krishnan and STRIDE for assistance with this study and access to patient data. STRIDE (Stanford Translational Research Integrated Database Environment) is a research and development project at Stanford University to create a standardsbased informatics platform supporting clinical and translational research. The project described was supported by the National Center for Research Resources and the National Center for Advancing Translational Sciences, National Institutes of Health, through grant UL1 TR001085. The content is solely the responsibility of the authors and does not necessarily represent the official views of the NIH.

Contributors QM, JC, and RD conceived the described experiments. DS acquired the University of California, San Francisco (UCSF) data. QM and YZ executed the experiments. QM, RD, JC and MJ interpreted the results. QM, MJ and JLH wrote the manuscript. QM, RD, MJ, JLH, JC, CB, DS, LS, UC, GF and YK revised the manuscript.

Funding Research reported in this publication was supported by the National Science Foundation under grant no 1549867.

Disclaimer The content is solely the responsibility of the authors and does not necessarily represent the official views of the National Science Foundation.

Competing interests All authors who have affiliations listed with Dascena (Hayward, California,USA) are employees or contractors of Dascena. CB reports receiving consulting fees from Dascena. $\mathrm{CB}, \mathrm{LS}, \mathrm{DS}$ and GF report receiving grant funding from Dascena.

Patient consent Not required.

Provenance and peer review Not commissioned; externally peer reviewed.

Data sharing statement No data obtained from UCSF, Stanford, Oroville Hospital, Cape Regional Medical Center or Bakersfield Heart Hospital in this study can be shared or made available for open access. Multiparameter Intelligent Monitoring inIntensive Care III (MIMIC-III) is a publicly available database. Please visit https:// mimic.physionet.org for information on using the MIMIC-III database.

Open Access This is an Open Access article distributed in accordance with the Creative Commons Attribution Non Commercial (CC BY-NC 4.0) license, which permits others to distribute, remix, adapt, build upon this work non-commercially, and license their derivative works on different terms, provided the original work is properly cited and the use is non-commercial. See: http://creativecommons.org/ licenses/by-nc/4.0/ (c) Article author(s) (or their employer(s) unless otherwise stated in the text of the article) 2018. All rights reserved. No commercial use is permitted unless otherwise expressly granted.

\section{REFERENCES}

1. Murphy SL, Xu J, Kochanek KD. Deaths: final data for 2010. Nat/ Vital Stat Rep 2013;61:1-17.

2. Angus DC, Linde-Zwirble WT, Lidicker J, et al. Epidemiology of severe sepsis in the United States: analysis of incidence, outcome, and associated costs of care. Crit Care Med 2001;29:1303-10.

3. Stevenson EK, Rubenstein AR, Radin GT, et al. Two decades of mortality trends among patients with severe sepsis: a comparative meta-analysis*. Crit Care Med 2014;42:625.

4. Pfuntner A, Wier LM, Steiner C. Costs for Hospital Stays in the United States, 2010: Statistical Brief \#146. In Healthcare Cost and Utilization Project (HCUP) Statistical Briefs:Cost H, Project U, Rockville; MDAgency for Healthcare Research and QualityUSA, 2006. https://www.ncbi.nlm.nih.gov/books/NBK121966/.

5. O'Brien J. The Cost of Sepsis. CDC Safe Healthcare Blog 2015 https://blogs.cdc.gov/safehealthcare/the-cost-of-sepsis/\#ref.

6. Gaieski DF, Edwards JM, Kallan MJ, et al. Benchmarking the incidence and mortality of severe sepsis in the United States. Crit Care Med 2013;41:1167-74.

7. Rivers E, Nguyen B, Havstad S, et al. Early goal-directed therapy in the treatment of severe sepsis and septic shock. N Engl J Med 2001;345:1368-77.

8. Nguyen HB, Corbett SW, Steele R, et al. Implementation of a bundle of quality indicators for the early management of severe sepsis and septic shock is associated with decreased mortality. Crit Care Med 2007;35:1105-12.

9. Kumar A, Roberts D, Wood KE, et al. Duration of hypotension before initiation of effective antimicrobial therapy is the critical determinant of survival in human septic shock. Crit Care Med 2006;34:1589-96.

10. Levy MM, et al. sccm/esicm/accp/ats/sis international sepsis definitions conference. Intensive Care Med 2001;2003:530-8.

11. Singer M, Deutschman CS, Seymour CW, et al. The Third International Consensus Definitions for Sepsis and Septic Shock (Sepsis-3). JAMA 2016;315:801-10.

12. Subbe CP, Slater A, Menon D, et al. Validation of physiological scoring systems in the accident and emergency department. Emerg Med J 2006;23:841-5.

13. Rangel-Frausto MS, Pittet D, Costigan M, et al. The natural history of the systemic inflammatory response syndrome (SIRS). A prospective study. JAMA 1995;273:117-23.

14. Vincent JL, Moreno R, Takala J, et al. The SOFA (Sepsis-related Organ Failure Assessment) score to describe organ dysfunction/ failure. On behalf of the Working Group on Sepsis-Related Problems of the European Society of Intensive Care Medicine. Intensive Care Med 1996;22:707-10.

15. Johnson AE, Pollard TJ, Shen L, et al. MIMIC-III, a freely accessible critical care database. Sci Data 2016;3:160035.

16. Lowe HJ, Ferris TA, Hernandez PM, et al. STRIDE--An integrated standards-based translational research informatics platform. AMIA Annu Symp Proc 2009;2009:391-5.

17. PostgreSQL Global Development Group. https://www.postgresql. org/ (5 Oct 2017).

18. Van Rossum G. The Python Language Reference Manual: Network Theory Ltd, 2003. https://www.python.org/.

19. Henry KE, Hager DN, Pronovost PJ, et al. A targeted real-time early warning score (TREWScore) for septic shock. Sci Trans/ Med 2015;7:299ra122.

20. Fullerton JN, Price CL, Silvey NE, et al. Is the Modified Early Warning Score (MEWS) superior to clinician judgement in detecting critical illness in the pre-hospital environment? Resuscitation 2012;83:557-62.

21. Ben-David S, Blitzer J, Crammer K, et al. A theory of learning from different domains. Mach Learn 2010;79:151-75.

22. Calvert JS, Price DA, Chettipally UK, et al. A computational approach to early sepsis detection. Comput Biol Med 2016;74:69-73.

23. Desautels T, Calvert J, Hoffman J, et al. Prediction of Sepsis in the Intensive Care Unit With Minimal Electronic Health Record Data: A Machine Learning Approach. JMIR Med Inform 2016;4:e28.

24. Calvert J, Desautels T, Chettipally U, et al. High-performance detection and early prediction of septic shock for alcohol-use disorder patients. Ann Med Surg 2016;8:50-5.

25. Calvert JS, Price DA, Barton CW, et al. Discharge recommendation based on a novel technique of homeostatic analysis. J Am Med Inform Assoc 2017;24:24-9. 
26. Calvert J, Mao Q, Rogers AJ, et al. A computational approach to mortality prediction of alcohol use disorder inpatients. Comput Biol Med 2016;75:74-9.

27. Calvert J, Mao Q, Hoffman JL, et al. Using electronic health record collected clinical variables to predict medical intensive care unit mortality. Ann Med Surg 2016;11:52-7.

28. Iwashyna TJ, Odden A, Rohde J, et al. Identifying patients with severe sepsis using administrative claims: patient-level validation of the angus implementation of the international consensus conference definition of severe sepsis. Med Care 2014;52:e39.

29. Gultepe E, Green JP, Nguyen H, et al. From vital signs to clinical outcomes for patients with sepsis: a machine learning basis for a clinical decision support system. J Am Med Inform Assoc 2014:21:315-25.

30. Brause R, Hamker F, Paetz J. Septic shock diagnosis by neural networks and rule based systems. In: Schmitt M, Teodorescu HN, Jain $\mathrm{A}$, eds. et alComputational intelligence techniques in medical diagnosis and prognosis. New York: Springer, 2002:323-56.

31. Thiel SW, Rosini JM, Shannon W, et al. Early Prediction of Septic Shock. J. Hosp. Med 2010;1:19-25.

32. Horng S, Sontag DA, Halpern Y, et al. Creating an automated trigger for sepsis clinical decision support at emergency department triage using machine learning. PLoS One 2017; 12:e0174708. 